\title{
THE KKM MAPS AND FIXED POINT THEOREMS IN CONVEX SPACES
}

\author{
POLLY W. SY AND SEHIE PARK
}

\begin{abstract}
From a general form of the celebrated Knaster-Kuratowski-Mazurkiewicz (simply, KKM) theorem, we deduce a new form of the Fan-Browder fixed point theorem, an approximate fixed point theorem, and the Himmelberg fixed point theorem.
\end{abstract}

In this paper, a generalization of the Knaster-Kuratowski-Mazurkiewicz theorem is applied to obtain a new non-compact form of the Fan-Browder fixed point theorem. This is used to obtain an approximate fixed point theorem, which readily implies the Himmelberg fixed point theorem. Finally, we note that most of the results in this paper are equivalent to the Brouwer fixed point theorem.

A multimap (or simply, a map) $F: X \multimap Y$ is a function from a set $X$ into the power set $2^{Y}$ of a set $Y$; that is, a function with the values $F(x) \subset Y$ for $x \in X$ and the fibers $F^{-}(y):=\{x \in X: y \in F(x)\}$ for $y \in Y$. For $A \subset X$, let $F(A):=\bigcup\{F(x): x \in A\}$.

For a set $D$, let $\langle D\rangle$ denote the set of nonempty finite subsets of $D$.

Let $X$ be a subset of a vector space and $D$ a nonempty subset of $X$. We call $(X, D)$ a convex space if $\operatorname{co} D \subset X$ and $X$ has a topology that induces the Euclidean topology on the convex hulls of any $N \in\langle D\rangle$; see [8], [9]. If $X=D$ is convex, then $X=(X, X)$ becomes a convex space in the sense of Lassonde [7]. If $X$ is compact, then the convex space $(X, D)$ is said to be compact. Every nonempty convex subset $X$ of a topological vector space is a convex space with respect to any nonempty subset $D$ of $X$, and the converse is known to be not true.

The following version of the Knaster-Kuratowski-Mazurkiewicz (simply, KKM) theorem for convex spaces is known.

Theorem 1. Let $(X, D)$ be a convex space and $F: D \multimap X$ a multimap such that

(1) $F(z)$ is open $[$ resp. closed $]$ for each $z \in D$; and

(2) $F$ is a KKM map (that is, co $N \subset F(N)$ for each $N \in\langle D\rangle$ ).

Received April 23, 2002.

2000 Mathematics Subject Classification. 47H04, 47H10, 52H07, 54C60, 54H25.

Key words and phrases. KKM maps, convex spaces, Fan-Browder fixed point theorem, Himmelberg fixed point theorem. 
Then $\{F(z)\}_{z \in D}$ has the finite intersection property. (More precisely, for any $N \in\langle D\rangle$, we have co $N \cap\left[\bigcap_{z \in N} F(z)\right] \neq \emptyset$.)

The closed version is essentially due to Fan [3] and the open version is motivated from the works of Kim [6] and Shih-Tan [16], who showed that the original KKM theorem holds for open valued KKM maps on a simplex. Later, Lassonde [8] showed that the closed and open versions of Theorem 1 can be derived from each other. More general versions of Theorem 1 were recently known; for example, see Park ([13]-[15]).

From Theorem 1, we deduce the following new result.

Theorem 2. Let $(X, D)$ be a convex space and $P: X \multimap D$ a multimap. If there exist $z_{1}, z_{2}, \ldots, z_{n} \in D$ and nonempty open [resp. closed] subsets $G_{i} \subset P^{-}\left(z_{i}\right)$ for each $i=1,2, \ldots, n$ such that $\operatorname{co}\left\{z_{1}, z_{2}, \ldots, z_{n}\right\} \subset \bigcup_{i=1}^{n} G_{i}$, then the map co $P: X \multimap X$ has a fixed point $x_{0} \in X$ (more precisely, there exists an $N \in\left\langle P\left(x_{0}\right)\right\rangle$ such that $x_{0} \in \operatorname{co} N \subset$ co $\left.P\left(x_{0}\right)\right)$.

Proof. Let $Y:=\operatorname{co}\left\{z_{1}, z_{2}, \ldots, z_{n}\right\}$ and $D^{\prime}:=\left\{z_{1}, z_{2}, \ldots, z_{n}\right\} \subset D$ and consider the convex space $\left(Y, D^{\prime}\right)$. Define a map $F: D^{\prime} \multimap Y$ by $F\left(z_{i}\right):=Y \backslash G_{i}$ for each $z_{i} \in D^{\prime}$. Then each $F\left(z_{i}\right)$ is closed [resp. open] in $Y$, and

$$
\bigcap_{i=1}^{n} F\left(z_{i}\right)=Y \backslash \bigcup_{i=1}^{n} G_{i}=Y \backslash Y=\emptyset .
$$

Therefore, the family $\{F(z)\}_{z \in D^{\prime}}$ does not have the finite intersection property, and hence, $F$ is not a KKM map by Theorem 1. Thus, there exists an $N \in\left\langle D^{\prime}\right\rangle$ such that $\operatorname{co} N \not \subset F(N)=\bigcup\left\{Y \backslash G_{i}: z_{i} \in N\right\}$. Hence, there exists an $x_{0} \in \operatorname{co} N$ such that $x_{0} \in G_{i} \subset P^{-}\left(z_{i}\right)$ for each $z_{i} \in N$; that is, $N \subset P\left(x_{0}\right)$. Therefore, $x_{0} \in \operatorname{co} N \subset \operatorname{co} P\left(x_{0}\right)$. This completes our proof.

The following well-known Fan-Browder fixed point theorem is a simple consequence of Theorem 2 .

Theorem 3. Let $K$ be a compact convex space and $T: K \multimap K$ a map such that

(1) $T(x)$ is a nonempty convex subset of $K$ for each $x \in K$; and

(2) $T^{-}(y)$ is open in $K$ for each $y \in K$.

Then there exists an $x_{0} \in K$ such that $x_{0} \in T\left(x_{0}\right)$.

Proof. Since each $T(x)$ is nonempty, $K$ has the open cover $\left\{T^{-}(y)\right\}_{y \in K}$. Since $K$ is compact, this cover has a finite subcover $\left\{T^{-}\left(z_{i}\right)\right\}_{i=1}^{n}$. Let $D=\left\{z_{1}, z_{2}, \ldots, z_{n}\right\}$ and define $P: K \multimap D$ by $P(x):=\{z \in D: z \in T(x)\}$ for each $x \in K$. Then $P^{-}\left(z_{i}\right)=T^{-}\left(z_{i}\right)$ is open for each $z_{i} \in D$, and $P(x) \subset T(x)$ for each $x \in K$, whence we have $\operatorname{co} P(x) \subset T(x)$ by (1). Put $G_{i}:=P^{-}\left(z_{i}\right)=T^{-}\left(z_{i}\right)$ for each $i=1,2, \ldots, n$. Then $\operatorname{co} P$ has a fixed point by Theorem 2 . 
Theorem 3 is essentially due to Browder [2] and a reformulation of Fan's geometric lemma [3] in the form of a fixed point theorem. Browder [2] applied Theorem 3 to a systematic treatment of the interconnections between multi-valued fixed point theorems, minimax theorems, variational inequalities, and monotone extension theorems. For further developments on generalizations and applications of Theorem 3, we refer to [11], [13], and [14].

The following theorem due to Lassonde [8] also follows from Theorem 2.

Theorem 4. Let $X$ be a convex space and $T: X \multimap X$ a multimap such that

(1) $T(x)$ is convex for each $x \in X$;

(2) $T^{-}(y)$ is closed for each $y \in X$; and

(3) there exists an $A \in\langle X\rangle$ such that $T(x) \cap A \neq \emptyset$ for each $x \in X$.

Then T has a fixed point.

Proof. Let $D:=A=\left\{z_{1}, z_{2}, \ldots, z_{n}\right\}$ and $P(x):=\{z \in D: z \in T(x)\}$ for each $x \in X$. Then each $P(x)$ is nonempty by (3) and $\operatorname{co} P(x) \subset T(x)$ for each $x \in X$ by (1). Moreover, $G_{i}:=P^{-}\left(z_{i}\right)=T^{-}\left(z_{i}\right)$ is closed for each $i$, by (2). Further, by (3), $X=\bigcup_{i=1}^{n} P^{-}\left(z_{i}\right)=\bigcup_{i=1}^{n} G_{i}$. Therefore, co $P$ has a fixed point by Theorem 2 .

Note that Kim [6] deduced particular forms of Theorem 4 from the Kakutani fixed point theorem.

The following examples show that Theorem 2 properly generalize Theorems 3 and 4 .

Examples 1. Let $X:=[0,10) \subset \mathbb{R}$ and $T: X \multimap X$ be defined by $T(x):=(x / 2,10)$ for $x \in X$. Then each $T(x)$ is nonempty convex. Moreover, $T^{-}(y)=[0,2 y)$ if $y<5$ and $T^{-}(y)=[0,10)$ if $y \geq 5$, and hence $T^{-}(y)$ is open for each $y \in X$. Note that $X$ is covered by a finite number of $T^{-}(y)$ 's and hence Theorem 2 with $X=D$ works.

2. Let $X:=[0,2] \subset \mathbb{R}$ and $D:=\{0,2\}$. Define $P: X \multimap D$ by $P(x)=\{2\}$ if $0 \leq x \leq$ $1, P(1)=\{0,2\}$ and $P(x)=\{0\}$ if $1<x \leq 2$. Then $X=\operatorname{co} D=[0,2]=P^{-}(0) \cup P^{-}(2)$ is the union of closed sets and Theorem 2 works.

For topological spaces $X$ and $Y$, a multimap $F: X \multimap Y$ is said to be lower [resp. upper] semi-continuous if for each open [resp. closed] set $B \subset Y$, the set $F^{-}(B)=\{x \in$ $X: F(x) \cap B \neq \emptyset\}$ is open [resp. closed] in $X$.

From Theorem 2, we obtain the following approximate fixed point theorem.

Theorem 5. Let $X$ be a convex subset of a topological vector space and $F: X \multimap X$ a lower [resp. upper] semi-continuous map such that (1) $F(x)$ is nonempty convex for each $x \in X$ and (2) $F(X)$ is totally bounded. Then for every open [resp. closed] convex neighborhood $V$ of the origin $O$ of $E$, there exists a point $x_{V} \in X$ such that

$$
F\left(x_{V}\right) \cap\left(x_{V}+V\right) \neq \emptyset .
$$


Proof. Since $F(X)$ is totally bounded, there exists a subset $D \in\langle X\rangle$ such that $F(X) \subset D+V$. Then, for any $x \in X,(F(x)-V) \cap D \neq \emptyset$. Define a map $P: X \multimap D$ by $P(x):=(F(x)-V) \cap D$ for $x \in X$. Then each $P(x)$ is nonempty. Note that for each $y \in D$, we have

$$
\begin{aligned}
P^{-}(y) & =\{x \in X: y \in P(x) \cap D\} \\
& =\{x \in X: y \in(F(x)-V) \cap D\} \\
& =\{x \in X: F(x) \cap(y+V) \neq \emptyset\} .
\end{aligned}
$$

If $F$ is lower semi-continuous and $V$ is open, then each $P^{-}(y)$ is open. If $F$ is upper semi-continuous and $V$ is closed, then each $P^{-}(y)$ is closed.

Note that for each $x \in X$, we have a $y \in D$ such that $x \in P^{-}(y)$. Therefore, $X=\bigcup_{y \in D} P^{-}(y)$. Hence, by Theorem 2, co $P: X \multimap X$ has a fixed point $x_{V} \in X$, that is, $x_{V} \in \operatorname{co} P\left(x_{V}\right) \subset\left(F\left(x_{V}\right)-V\right) \cap X$, which readily implies $F\left(x_{V}\right) \cap\left(x_{V}+V\right) \neq \emptyset$.

Fan [4] obtained a slightly different version of Theorem 5 for a lower semi-continuous multimap $F: X \multimap E$, where $X$ is a convex subset of a locally convex Hausdorff topological vector space $E$.

Lassonde [8] obtained Theorem 5 for a compact map $F: X \multimap X$ using a particular form of Theorem 2. We followed his method in the proof of Theorem 5 .

A more general result including Theorem 5 and Theorems of Fan and Lassonde is due to Park ([12], Theorem 3) (where Hausdorffness is redundant) with a different proof.

From the upper semi-continuous case of Theorem 5, we deduce the well-known Himmelberg fixed point theorem [5] as follows. We give its proof for completeness.

Theorem 6. Let $X$ be a convex subset of a locally convex Hausdorff topological vector space $E$ and $F: X \multimap X$ a compact upper semi-continuous map having nonempty closed convex values. Then $F$ has a fixed point $x_{0} \in X$.

Proof. Since $E$ is locally convex, each neighborhood $U$ of $O$ contains a closed convex neighborhood of $O$. Therefore, by Theorem 4, there exist $x_{U}, y_{U} \in X$ such that $y_{U} \in$ $F\left(x_{U}\right)$ and $y_{U} \in x_{U}+U$. Since $F(X)$ is relatively compact in $X$, we may assume that the net $\left\{y_{U}\right\}$ converges to some $x_{0} \in X$. Since $E$ is Hausdorff, the net $\left\{x_{U}\right\}$ also converges to $x_{0}$. Because $F$ is upper semi-continuous with closed values, the graph of $F$ is closed in $X \times F(X)$ and hence we have $x_{0} \in F\left(x_{0}\right)$. This proves the theorem.

Note that Himmelberg's original proof is based on the Kakutani fixed point theorem. Theorem 6 includes historically well-known theorems due to Brouwer, Schauder, Tychonoff, Kakutani, Hukuhara, Bohnenblust and Karlin, Fan and Glicksberg. Moreover, there have appeared a number of another generalizations of the Himmelberg theorem; see [11]. 
Until now, we deduced all of our results from the KKM theorem, which is known to be equivalent to the Brouwer fixed point theorem. Therefore, in certain sense, all of our above results are equivalent to the Brouwer theorem.

Finally, from Theorem 5, we have the following.

Theorem 7. Let $X$ be a convex subset of a topological vector space, and $F: X \multimap X$ a multimap such that

(1) $F(x)$ is nonempty convex for each $x \in X$;

(2) $F^{-}(y)$ is open for each $y \in X$; and

(3) $F(X)$ is totally bounded.

Then for any convex neighborhood $V$ of $O$ in $E$, there exists a point $x_{V} \in X$ such that $F\left(x_{V}\right) \cap\left(x_{V}+V\right) \neq \emptyset$.

Proof. Note that $F$ is simply lower semi-continuous.

Theorem 7 improves Park ([12], Corollary 7), which is closely related to the Ben-ElMechaiekh conjecture [1], that is, Theorem 3 would hold if we assume the map $T: K \multimap$ $K$ is compact instead of the compactness of $K$. This is not resolved yet and a number of partial solutions were given in [10].

\section{Acknowledgement}

This paper was written while the second author was visiting the University of the Philippines, Diliman, Quezon City from 6 January 2002 to 5 February 2002. He would like to express his gratitude to the colleagues of the Department of Mathematics there for their kind hospitality.

\section{References}

[1] H. Ben-El-Mechaiekh, Fixed points for compact set-valued maps, Q and A in General Topology 10(1992), 153-156.

[2] F. E. Browder, The fixed point theory of multi-valued mappings in topological vector spaces, Math. Ann. 177(1968), 283-301.

[3] Ky Fan, A generalization of Tychonoff's fixed point theorem, Math. Ann. 142(1961), 305-310.

[4] Ky Fan, Covering properties of convex sets and fixed point theorems in topological vector spaces, Symposium on Infinite Dimensional Topology, Ann. of Math. Studies, No. 69, 79-92, Princeton University Press, 1972.

[5] C. J. Himmelberg, Fixed points of compact multifunctions, J. Math. Anal. Appl. 38(1972), 205-207.

[6] W. K. Kim, Some applications of the Kakutani fixed point theorem, J. Math. Anal. Appl. 121(1987), 119-122.

[7] M. Lassonde, On the use of KKM multifunctions in fixed point theory and related topics, J. Math. Anal. Appl. 97(1983), 151-201. 
[8] M. Lassonde, Sur le principe KKM, C. R. Acad. Sci. Paris, Série I, 310(1990), 573-576.

[9] Sehie Park, Foundations of the KKM Theory via coincidences of composites of upper semicontinuous maps, J. Korean Math. Soc. 31(1994), 493-519.

[10] Sehie Park, Remarks on a fixed point problem of Ben-El-Mechaiekh, in: Nonlinear Analysis and Convex Analysis (Proc. NACA'98, Niigata, Japan, Jul. 28-31, 1998), 79-86, World Sicentific, Singapore, 1999.

[11] Sehie Park, Ninety years of the Brouwer fixed point theorem, Vietnam J. Math. 27(1999), 187-222.

[12] Sehie Park, The Knaster-Kuratowski-Mazurkiewicz theorem and almost fixed points, Topological Methods in Nonlinear Anal., J. of the Julius Schauder Center, 16(2000), 195-200.

[13] Sehie Park, Elements of the KKM theory for generalized convex spaces, Korean J. Comp. Appl. Math. 7(2000), 1-28.

[14] Sehie Park, Remarks on topologies of generalized convex spaces, Nonlinear Funct. Anal. Appl. 5(2000), 67-79.

[15] Sehie Park, Fixed point theorems in locally G-convex spaces, Nonlinear Anal. 48(2002), 869-879.

[16] M.-H. Shih and K.-K. Tan, Covering theorems of convex sets related to fixed point theorems, in : Nonlinear and Convex Analysis (Proc. in Honor of Ky Fan), 235-244, Marcel Dekker, Inc., New York-Basel, 1987.

Department of Mathematics, College of Science, University of the Philippines, Diliman, Quezon City, Philippines.

E-mail: pweesy@i-manila.com.ph

School of Mathematical Sciences, Seoul National University, Seoul 151-747, Korea.

E-mail: spark@math.snu.ac.kr 